\section{COST-EFFECTIVENESS ANALYSIS FOR THE INTEGRATED CARE FOR ADVANCED RESPIRATORY DISORDER (ICARE) PROGRAM - A MULTIDISCIPLINARY PALLIATIVE REHABILITATION PROGRAM FOR CHRONIC LUNG DISEASE IN A COMMUNITY HOSPITAL}

LM Teo, HY Neo. Lee Kong Chian School of Medicine, Tan Tock Seng Hospital, Ren Ci Community Hospital

\subsection{6/spcare-2020-PCC.14}

Introduction Patients with chronic breathlessness suffer high symptom burden and unmet needs, driving repeated hospitalizations. ICARE program, based on a novel construct of 'Palliative Rehabilitation,' integrates early palliative care with postexacerbation, inpatient rehabilitation for patients with advanced non-malignant lung diseases. This study aims to examine ICARE's impact on healthcare resource utility, as well as secondary clinical and functional outcomes.

Methods This study compared the number of admissions and total length of stay (TLOS) 6-months pre-enrolment and postdischarge from ICARE. Formal healthcare cost is calculated using daily unit costs provided by Ministry of Health. Functional improvement before-and-after ICARE are measured via 6 minute-walk test (6MWT) and Modified Barthel Index (MBI). Clinical issues are identified and tracked using an assessment template.

Results 88 patients were eligible for analysis. There were significant reductions in acute-hospital TLOS (mean 14.0 days, $<0.05$ ) and number of admissions (mean 1.17, <0.05). By projection, reduction in TLOS accrued to an annual 2464 bed-days saved for the tertiary hospital. Net cost deficit was $\$ 581$ per patient. Subgroup analysis revealed patients with recurrent admissions 6-months prior to ICARE $(\mathrm{N}=44)$ generated annual savings of $\$ 308,949$, while 1-time admitters $(\mathrm{N}=44)$ incurred deficit of $\$ 410,810$. Notably, 1-time admitters had longer LOS in their index tertiary hospital admission and had poorer 6MWT and MBI on enrolment, suggesting more severe exacerbations with greater deconditioning. Functionally, significant improvements in 6MWT (median $30 \mathrm{~m}$, $<0.05$ ) and MBI (median 12.5points, <0.05) were observed. $76.5 \%$ clinical issues identified were improved/resolved.

Discussion ICARE leads to a cost deficit of \$581/patient, equivalent to only an additional half-a-day stay in tertiary hospital. Considering that patients functionally were not fit for discharge at point of enrolment into ICARE, this study demonstrates that ICARE is potentially cost-saving, particularly for recurrent admitters. ICARE also alleviates tertiary hospital bed-occupancy rate while improving clinical and functional outcomes.

\section{A STROKE AHEAD: PALLIATIVE CARE IN-REACH WORK ON THE STROKE WARDS}

Claire Leyland, Jeena Ackroyd, Oliver Hutchinson. Calderdale and Huddersfield NHS Foundation Trust

\subsection{6/spcare-2020-PCC.15}

Background The Intercollegiate Stroke Working Party (2016) outlined that services providing acute and long-term care for people with stroke should provide high quality end-of-life care. Difficulties occur in recognising dying when the trajectory of the disease is not easily predicted.
Method A project was developed which involved a band 7 CNS in Palliative care having 15 hours a week designated to the 4 stroke wards for a year. The model was of in-reach work. Data was collected prospectively for patients referred to the CNS using a pro forma. Information was also obtained by identifying patients who were diagnosed with a stroke who had a palliative care code. A survey was also developed to assess the impact of the project on ward staff.

Results 113 patients were seen by the project CNS over a 12 month period. The average Karnofsky Performance Score (KPS) of patients seen was 20. $80 \%$ of patients lacked capacity.

$38 \%$ referrals were related to managing patients in their last few days of their life.

$66 \%$ patients died in hospital but over a third were discharged with CNS support. $73 \%$ required symptom management. $66 \%$ involved supporting the stroke team with decision making and $42 \%$ involved facilitating family discussions. $16 \%$ patients were referred with artificial nutrition, but only $1 \%$ patients died with nutrition in situ. Satisfaction with palliative care input increased from $43 \%$ to $92 \%$

Conclusions Regular palliative input to the stroke wards supported complex decision making for patients who often lacked capacity. Key roles included supporting staff to recognise dying and stopping artificial nutrition. Initiating discharge planning was also an important aspect and has led to the development of a discharge coordinator specifically for palliative care patients. The in-reach model was valued by staff.

\section{Free papers 16 - 18: future}

\section{TO DRIP OR NOT TO DRIP: INADEQUATE EVIDENCE TO GUIDE PRACTICE. A SYSTEMATIC LITERATURE REVIEW AND NARRATIVE SYNTHESIS}

Arjun Kingdon, Stephen Barclay, Anna Spathis. University of Cambridge

\subsection{6/spcare-2020-PCC.16}

Background The impact of clinically assisted hydration on quality of life or survival in the last days of life is not known. A previous systematic review evaluated only trial data, mostly from studies focusing on patients in the last weeks of life, and found insufficient evidence to draw firm conclusions. This issue is often highly emotive. Practice varies significantly worldwide.

Aim To review the published evidence concerning assisted hydration in the final days of life, focusing on symptoms, side effects, survival, quality of life, and the views of patients and families.

Design Systematic literature review and narrative synthesis of studies using a range of methods. Databases were searched up to September 2019 alongside reference and journal hand searches. Research quality was appraised using Gough's 'Weight of Evidence' framework.

Results The search yielded 4053 studies. Of the 27 papers included in the synthesis, only one study was judged to be of high quality and relevance. Most studies demonstrated little or no impact of clinically assisted hydration on quality of life, symptoms, or survival: many were poorly designed or underpowered. Of the ten studies investigating delirium, four (including the only high quality study) suggested an association 